\title{
Absence of DICER1, CTCF, RPL22, DNMT3A, TRRAP, $I D H 1$ and $I D H 2$ hotspot mutations in patients with various subtypes of ovarian carcinomas
}

\author{
YANG ZOU ${ }^{1,2^{*}}$, MEI-ZHEN HUANG ${ }^{1-3^{*}}$, FA-YING LIU ${ }^{1,2}$, BI-CHENG YANG ${ }^{1}$, LI-QUN WANG ${ }^{1}$, FENG WANG ${ }^{1,2}$, \\ XIAO-HONG YU ${ }^{4}$, LEI WAN ${ }^{1}$, XI-DI WAN ${ }^{1}$, XIAO-YUN XU ${ }^{1-3}$, WEI LI ${ }^{1-3}$, OU-PING HUANG ${ }^{1,2}$ and MING HE ${ }^{5}$ \\ ${ }^{1}$ Key Laboratory of Women's Reproductive Health of Jiangxi Province; ${ }^{2}$ Central Laboratory, Jiangxi Provincial Maternal \\ and Child Health Hospital, Nanchang, Jiangxi 330006; ${ }^{3}$ Graduate School of Nanchang University, Nanchang, Jiangxi 330031; \\ ${ }^{4}$ Department of Pathology, Jiangxi Provincial Maternal and Child Health Hospital; ${ }^{5}$ Department of Pharmacology and \\ Molecular Therapeutics, Nanchang University School of Pharmaceutical Science, Nanchang, Jiangxi 330006, P.R. China
}

Received September 22, 2014; Accepted October 24, 2014

DOI: $10.3892 /$ br. 2014.378

\begin{abstract}
Cancer is caused by multiple genetic alterations within cells. Recently, large-scale sequencing has identified frequent ribonuclease type III (DICERI), CCCTC-binding factor $(C T C F)$, ribosomal protein L22 (RPL22), DNA (cytosine-5-)-methyltransferase $3 \alpha$ (DNMT3A), transformation/transcription domain-associated protein (TRRAP), isocitrate dehydrogenase $(I D H) 1$ and $I D H 2$ hotspot mutations in diverse types of cancer. However, it remains largely unknown whether these mutations also exist in ovarian carcinomas. In the present study, a collection of 251 patients with distinct subtypes of ovarian carcinomas were recruited and sequenced for the presence of these hotspot mutations. However, no mutations in the seven genes were detected in the samples. These negative results, together with certain recent reports, indicate that the hotspot mutations in the $C T C F$, RPL22, DNMT3A, TRRAP, IDH1 and IDH2 genes may not be actively involved in the carcinogenesis of ovarian carcinoma. Of note, the DICER1 mutation frequency in Sertoli-Leydig cell tumor in the present study was significantly lower compared to prior observation, and therefore, it is speculated that this discrepancy may be mainly due to the small sample
\end{abstract}

Correspondence to: Professor Ou-Ping Huang, Key Laboratory of Women's Reproductive Health of Jiangxi Province and Central Laboratory, Jiangxi Provincial Maternal and Child Health Hospital, 318 Bayi Road, Nanchang, Jiangxi 330006, P.R. China

E-mail: huangouping@gmail.com

Professor Ming He, Department of Pharmacology and Molecular Therapeutics, Nanchang University School of Pharmaceutical Science, 461 Bayi Road, Nanchang, Jiangxi 330006, P.R. China E-mail: jxhm56@hotmail.com

${ }^{*}$ Contributed equally

Key words: hotspot mutations, absence, ovarian carcinoma size analyzed in the study. In addition, among these samples, frequent polymerase (DNA directed) $\varepsilon$, catalytic subunit (POLE1) and ring finger protein 43 (RNF43) mutations were identified in endometrioid and mucinous ovarian carcinomas, respectively; thus DICER1, CTCF, RPL22, DNMT3A, TRRAP, $I D H 1$ and $I D H 2$ hotspot mutations may not play synergistic roles with POLE1 or RNF43 mutations in the carcinogenesis of endometrioid or mucinous ovarian carcinomas.

\section{Introduction}

The current understanding of human malignancy is that it mainly arises due to the accumulation of multiple genetic alterations, transforming normal cells into malignant cells $(1,2)$. Of these genetic alterations, a myriad of genomic mutation data derived from a high-throughput DNA sequencing technique provided a unique opportunity to profile the mutation spectra underlying human cancers and a large number of significant functional mutations in multiple genes were identified in diverse types of cancer $(1,3,4)$. These genes can be defined as oncogenes or tumor suppressor genes and are being used as molecular markers for diagnosis, staging and prognosis of human cancers $(5,6)$.

Ovarian carcinoma constitutes a heterogeneous group of malignancies with significantly different clinical expression, pathological characteristics and genetic etiology $(7,8)$. However, the majority of ovarian carcinomas shared certain common genetic alterations, such as frequent tumor protein p53 (TP53) and PIK3CA, catalytic subunit $\alpha$ mutations $(9,10)$, and patients also exhibited subtype-specific mutations (11-13), which are possibly essential for the differential clinical expression and molecular-targeted therapy in ovarian carcinomas $(14,15)$. These observations emphasized the requirement to identify novel subtype-specific molecular genetic aberrations in ovarian carcinomas.

Recently, large-scale sequencing has identified frequent mutations of the ribonuclease type III (DICERI) gene in Sertoli-Leydig cell tumors of the ovary (3), CCCTC-binding factor $(C T C F)$ gene in transient abnormal myelopoiesis (16) and 
Table I. Mutation frequencies of ribonuclease type III (DICERI), CCCTC-binding factor (CTCF), ribosomal protein L22 (RPL22), DNA (cytosine-5-)-methyltransferase $3 \alpha$ (DNMT3A), transformation/transcription domain-associated protein (TRRAP), isocitrate dehydrogenase $(I D H) 1$ and $I D H 2$ hotspot mutations in 251 Chinese patients with ovarian carcinomas.

\begin{tabular}{|c|c|c|c|c|c|c|c|c|c|c|c|c|}
\hline Subtype/gene & No. & $\begin{array}{c}\text { DICER1 } \\
\text { p.1705- } \\
1709\end{array}$ & $\begin{array}{c}\text { DICER1 } \\
\text { p.1810- } \\
1813\end{array}$ & $\begin{array}{c}C T C F \\
\text { p.T204fs* }\end{array}$ & $\begin{array}{c}R P L 22 \\
\text { c.43delA }\end{array}$ & $\begin{array}{l}\text { DNMT3A } \\
\text { p.R882 }\end{array}$ & $\begin{array}{l}\text { TRRAP } \\
\text { p.S722 }\end{array}$ & $\begin{array}{l}\text { IDHI } \\
\text { p.R132 }\end{array}$ & $\begin{array}{c}I D H 2 \\
\text { p.R140 }\end{array}$ & $\begin{array}{c}I D H 2 \\
\text { p.R172 }\end{array}$ & $\begin{array}{c}R N F 43 \\
\text { p.I48V and } \\
\text { p.R40fs* } 11^{\mathrm{a}}\end{array}$ & $\begin{array}{c}\text { POLE1 } \\
\text { p.S297F }\end{array}$ \\
\hline \multicolumn{13}{|l|}{ Epithelial } \\
\hline Serous & 76 & $0 / 76$ & $0 / 76$ & $0 / 72$ & $0 / 75$ & $0 / 74$ & $0 / 75$ & $0 / 73$ & $0 / 74$ & $0 / 74$ & $0 / 74$ & $0 / 74$ \\
\hline Clear cell & 43 & $0 / 43$ & $0 / 43$ & $0 / 42$ & $0 / 43$ & $0 / 43$ & $0 / 43$ & $0 / 42$ & $0 / 41$ & $0 / 41$ & $0 / 41$ & $0 / 41$ \\
\hline Endometrioid & 37 & $0 / 37$ & $0 / 37$ & $0 / 35$ & $0 / 35$ & $0 / 36$ & $0 / 37$ & $0 / 35$ & $0 / 37$ & $0 / 37$ & $0 / 37$ & $3 / 37$ \\
\hline Mucinous & 15 & $0 / 15$ & $0 / 15$ & $0 / 14$ & $0 / 15$ & $0 / 15$ & $0 / 15$ & $0 / 14$ & $0 / 15$ & $0 / 15$ & $2 / 15$ & $0 / 15$ \\
\hline Undifferentiated & 3 & $0 / 3$ & $0 / 3$ & $0 / 3$ & $0 / 3$ & $0 / 3$ & $0 / 3$ & $0 / 3$ & $0 / 3$ & $0 / 3$ & $0 / 3$ & $0 / 3$ \\
\hline Unclassified & 4 & $0 / 4$ & $0 / 4$ & $0 / 3$ & $0 / 4$ & $0 / 4$ & $0 / 4$ & $0 / 4$ & $0 / 4$ & $0 / 4$ & $0 / 4$ & $0 / 4$ \\
\hline Transitional cell & 3 & $0 / 3$ & $0 / 3$ & $0 / 3$ & $0 / 3$ & $0 / 3$ & $0 / 3$ & $0 / 3$ & $0 / 3$ & $0 / 3$ & $0 / 3$ & $0 / 3$ \\
\hline Mixed & 2 & $0 / 2$ & $0 / 2$ & $0 / 2$ & $0 / 2$ & $0 / 2$ & $0 / 2$ & $0 / 2$ & $0 / 2$ & $0 / 2$ & $0 / 2$ & $0 / 2$ \\
\hline \multicolumn{13}{|l|}{ Non-epithelial } \\
\hline \multicolumn{13}{|l|}{ Germ cell tumor } \\
\hline Yolk sac & 11 & $0 / 11$ & $0 / 11$ & $0 / 10$ & $0 / 11$ & $0 / 11$ & $0 / 10$ & $0 / 11$ & $0 / 11$ & $0 / 11$ & $0 / 11$ & $0 / 11$ \\
\hline Dysgerminoma & 7 & $0 / 7$ & $0 / 7$ & $0 / 7$ & $0 / 7$ & $0 / 7$ & $0 / 7$ & $0 / 6$ & $0 / 6$ & $0 / 6$ & $0 / 6$ & $0 / 6$ \\
\hline Teratoma & 9 & $0 / 9$ & $0 / 9$ & $0 / 8$ & $0 / 9$ & $0 / 9$ & $0 / 9$ & $0 / 8$ & $0 / 9$ & $0 / 9$ & $0 / 9$ & $0 / 9$ \\
\hline Mixed & 6 & $0 / 6$ & $0 / 6$ & $0 / 6$ & $0 / 6$ & $0 / 6$ & $0 / 6$ & $0 / 6$ & $0 / 6$ & $0 / 6$ & $0 / 6$ & $0 / 6$ \\
\hline \multicolumn{13}{|l|}{$\begin{array}{l}\text { Sex cord-stromal } \\
\text { tumor }\end{array}$} \\
\hline Granulosa cell & 16 & $0 / 16$ & $0 / 16$ & $0 / 14$ & $0 / 16$ & $0 / 16$ & $0 / 16$ & $0 / 15$ & $0 / 16$ & $0 / 16$ & $0 / 16$ & $0 / 16$ \\
\hline Sertoli-Leydig & 2 & $0 / 2$ & $0 / 2$ & $0 / 2$ & $0 / 2$ & $0 / 2$ & $0 / 2$ & $0 / 2$ & $0 / 2$ & $0 / 2$ & $0 / 2$ & $0 / 2$ \\
\hline Krukenberg tumors & 17 & $0 / 17$ & $0 / 17$ & $0 / 16$ & $0 / 16$ & $0 / 17$ & $0 / 17$ & $0 / 15$ & $0 / 17$ & $0 / 17$ & $0 / 17$ & $0 / 17$ \\
\hline
\end{tabular}

${ }^{\mathrm{a}, \mathrm{b}}$ As per references $(25,26) ; P O L E 1$, polymerase (DNA directed) $\varepsilon$, catalytic subunit; $R N F 43$, ring finger protein 43 .

endometrial cancer (17), ribosomal protein L22 (RPL22) gene in endometrial cancer (18), DNA (cytosine-5-)-methyltransferase $3 \alpha$ (DNMT3A) gene in hematological malignancies (4), the transformation/transcription domain-associated protein $(T R R A P)$ gene in melanoma (19) and isocitrate dehydrogenase 1 and 2 (IDHI and IDH2) genes in gliomas $(1,20)$ and acute myeloid leukemia (AML) (21), respectively. Some of these mutations were closely associated with cancer progression $(22)$ and prognosis $(23,24)$.

Thus far, the mutation statuses of DICER1, CTCF, RPL22, DNMT3A, TRRAP, IDH1 and IDH2 mutational hotspots in ovarian carcinomas remain largely unknown. One critical concern in cancer genetics is whether those cancer-associated mutations identified in one type of cancer are also common in other types of cancer. Therefore, a cohort of 251 Chinese patients with distinct subtypes of ovarian carcinomas was recruited in the present study to examine whether the hotspot mutations in these genes also existed in these samples.

\section{Materials and methods}

Sample collection. The study included 251 archival formalin-fixed, paraffin-embedded (FFPE) tissues with various subtypes of ovarian carcinoma recruited from the Jiangxi Provincial Maternal and Child Health Hospital (Nanchang, Jiangxi, China). Only those patients with $>70 \%$ of neoplastic cells were recruited in the study. The sample cohort contained 76 ovarian serous carcinoma, 43 ovarian clear cell carcinoma, 37 ovarian endometrioid carcinoma, 33 ovarian germ cell tumor, 15 mucinous ovarian carcinoma, 18 ovarian sex cord-stromal tumor, 12 other rare subtypes and 17 Krukenberg tumor, and the available clinical data was as described previously $(25,26)$ and in Table I. Informed consent conforming to the tenets of the Declaration of Helsinki was obtained from each patient prior to the study. The Institutional Review Boards of the Jiangxi Provincial Maternal and Child Health Hospital approved the study.

Mutation analysis of the DICER1, CTCF, RPL22, DNMT3A, TRRAP, IDH1 and IDH2 genes. The Omega FFPE DNA kit (Omega Bio-tek Inc., Doraville, GA, USA) was used to isolate the DNA from the FFPE tissues. The polymerase chain reaction (PCR) primers were as summarized previously $(25,27)$ and are shown in Table II. PCR reactions were performed in a total volume of $25 \mu \mathrm{l}$, containing $50 \mathrm{ng}$ genomic DNA, 2 units of LA Taq DNA Polymerase (Takara Biotechnology Dalian Co. Ltd., Liaoning, China), $300 \mu \mathrm{M}$ of each dNTP and $0.2 \mu \mathrm{M}$ of each primer. The amplification reaction was performed in a Thermal Cycler 2720 (Applied Biosystems, Foster City, CA, USA) and employed one denaturation cycle of $94^{\circ} \mathrm{C}$ for $3 \mathrm{~min}, 35$ amplification cycles of $94^{\circ} \mathrm{C}$ for $30 \mathrm{sec}$, $50-60^{\circ} \mathrm{C}$ (Table II) $(25,27)$ for $20 \mathrm{sec}$ and $72^{\circ} \mathrm{C}$ for $30 \mathrm{sec}$, 
Table II. Primers for the mutational analysis of the ribonuclease type III (DICERl), CCCTC-binding factor $(C T C F)$, ribosomal protein L22 (RPL22), DNA (cytosine-5-)-methyltransferase $3 \alpha$ (DNMT3A), transformation/transcription domain-associated protein $(T R R A P)$, isocitrate dehydrogenase $(I D H) 1$ and IDH2 genes.

\begin{tabular}{|c|c|c|c|c|c|c|}
\hline Gene & Target regions & $\begin{array}{l}\text { Amplicon, } \\
\text { bp }\end{array}$ & $\begin{array}{c}\text { Sample } \\
\text { detected }\end{array}$ & $\begin{array}{c}\text { Annealing, } \\
{ }^{\circ} \mathrm{C}\end{array}$ & Forward primer $\left(5^{\prime}-3^{\prime}\right)$ & Reverse primer $\left(5^{\prime}-3^{\prime}\right)$ \\
\hline DICERI & p.E1705-D1709 & 159 & $251 / 251$ & 55 & CGGATCCCCTCAGATTGTTA & CGATGCAAAGATGGTGTTGT \\
\hline DICERI & p.D1810-E1813 & 171 & $251 / 251$ & 55 & TGGCCTTTTTGCTTACAAGTC & TGCCAGACTGTCTCCAGTGA \\
\hline CTCF & p.T204fs* & 212 & $237 / 251$ & 56 & GTTAAAGTGGGGGCCAATG & AGCAGACCCTCCTGCTGTT \\
\hline RPL22 & c. $43 \mathrm{del} \mathrm{A}$ & 190 & $247 / 251$ & 60 & TCTTGTTTTTCCGACTGACTGA & CCGAGTGGCAATAAGGATGT \\
\hline DNMT3A & p.R882 & 177 & $248 / 251$ & 52 & TGCCCTCTCTGCCTTTTCT & CCATGTCCCTTACACACACG \\
\hline TRRAP & p.S722 & 183 & $249 / 251$ & 52 & TCTGCTCTGTTTGCTACGAT & GCACTACTTAGATTAAATGGAC \\
\hline IDH1 & p.R132 & 269 & $239 / 251$ & 50 & TGCTGCAGAAGCTATAAAGAAG & GCAAAATCACATTATTGCCAAC \\
\hline$I D H 2$ & p.R140 and p.R172 & 209 & $246 / 251$ & 50 & GCTGCAGTGGGACCACTATT & ACCCTGGCCTACCTGGTC \\
\hline
\end{tabular}

bp, base pair.
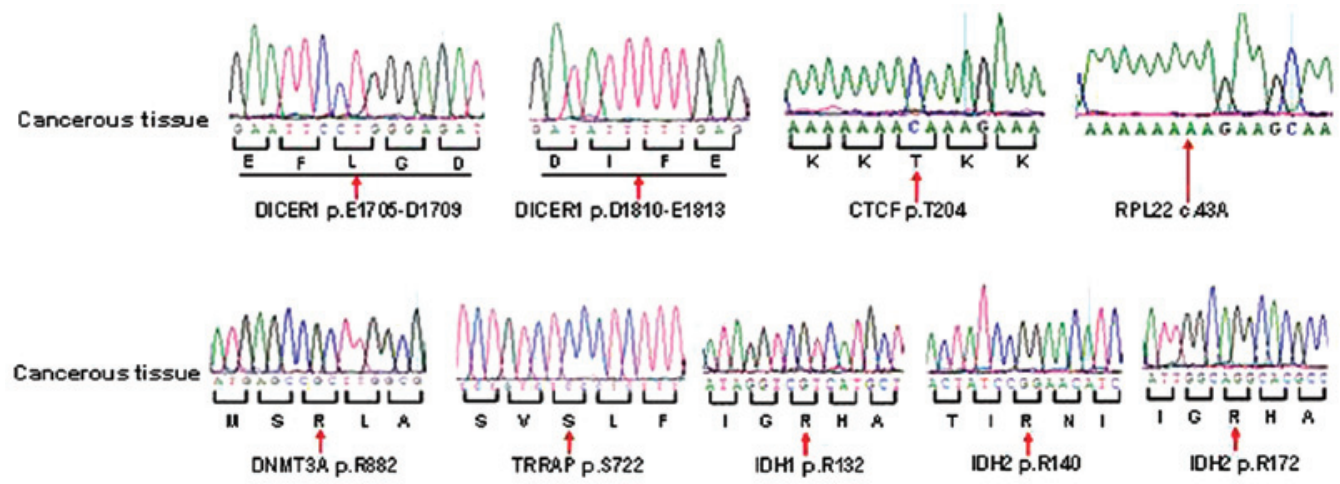

Figure 1. Representative sequencing electropherograms of the ribonuclease type III (DICER1), CCCTC-binding factor (CTCF), ribosomal protein L22 (RPL22), DNA (cytosine-5-)-methyltransferase $3 \alpha$ (DNMT3A), transformation/transcription domain-associated protein (TRRAP), isocitrate dehydrogenase $(I D H) 1$ and $I D H 2$ genes in Chinese patients with ovarian carcinomas.

with one final extension cycle of $72^{\circ} \mathrm{C}$ for $10 \mathrm{~min}$. The PCR products were purified and sequenced with an ABI 3730 DNA sequencer (Applied Biosystems). DNA sequence analyses were performed with the DNASTAR package software (DNASTAR Inc., Madison, WI, USA).

\section{Results and Discussion}

The available clinical data of these patients are as described previously $(25,26)$. In the present study, a total of 251 Chinese samples with distinct subtypes of ovarian carcinoma were screened for the presence of potential hotspot mutations in the DICER1, CTCF, RPL22, DNMT3A, TRRAP, IDH1 and IDH2 genes. However, no mutations in these genes were detected in the 251 samples (Table I and Fig. 1).

Previous studies have found frequent DICER 1 p.E1705-D1709 and p.D1810-E1813 mutations in Sertoli-Leydig cell tumors $(3,28)$. However, no DICERl mutations were detected in the two patients with Sertoli-Leydig cell tumors. Therefore, it can be speculated that this discrepancy may be caused mainly by the small sample size of the Sertoli-Leydig cell tumors analyzed in the present study. In addition, DICERI mutations were not identified in other subtypes of ovarian carcinomas in the samples, which is consistent with previous large-scale sequencing results in which the DICER 1 hotspot mutations were absent in 12 mucinous (29) or 316 serous ovarian carcinomas (9). Collectively, these results indicated that the DICER 1 hotspot mutations may not be actively involved in the pathogenesis of ovarian carcinoma, except for Sertoli-Leydig cell tumors.

CTCF p.T204fs* and RPL22 c.43delA mutations have been observed frequently in endometrial carcinoma in previously studies $(17,18)$. Considering the fact that ovarian carcinoma have certain overlapped genetic aberrations with endometrial cancer, such as frequent TP53 $(9,30)$ and polymerase (DNA directed) $\varepsilon$, catalytic subunit (POLE1) mutations $(26,30)$, we hypothesized that ovarian carcinomas may also harbor these mutations. However, neither CTCF p.T204fs* nor RPL22 c.43delA mutations were identified in the samples in the present study. The absence of the CTCF and RPL22 mutations in ovarian cancer in a previous study (29) and the present study suggested that the CTCF and RPL22 hotspot mutations may play an extremely limited role in the pathogenesis of ovarian cancer.

Prevalent TRRAP p.S722 mutation was initially identified in melanomas in a whole-exome sequencing study (19). Subsequent extended studies failed to identify these mutations in thyroid cancer (31) or splenic marginal zone lymphoma (32). In the present study, no TRRAP p.S722 mutations were detected 
in our ovarian cancer patients with distinct subtypes. Also, TRRAP p.S722 mutations were not found in 12 mucinous (29) or 316 serous ovarian carcinomas (9). These negative results led us to speculate that TRRAP p.S722 mutations may not play a crucial role in the malignant transformation of ovarian carcinoma.

DNMT3A p.R882 mutations were identified almost exclusively in hematological malignancies, including AML (33), acute lymphoblastic leukemia (34) and myelodysplastic syndromes (35), and are generally infrequent or absent in some solid tumors $(9,29,36)$. DNMT3A p.R882 mutations were not detected in the 251 samples with distinct subtypes of ovarian carcinoma. Similarly, whole-exome sequencing studies suggested that DNMT3A p.R882 mutations were absent in 12 mucinous (29) or 316 serous ovarian carcinomas (9). Taken together, the absence of DNMT3A p.R882 mutations in ovarian carcinoma analyzed in the present study and in previous studies $(9,29)$ indicated that DNMT3A p.R882 mutations may be infrequent in ovarian carcinoma.

Frequent $I D H 1$ p.R132, and IDH2 p.R140 and p.R172 mutations were identified in the central nervous system tumors and AML $(1,20,27)$. However, no IDH1 or IDH2 mutations were detected in the present samples. Similar results were observed in previous studies in which IDH1 p.R132 mutations were not detected in 168 ovarian carcinomas or 8 ovarian cancer cell lines (20,37-39). In addition, $I D H 1$ and $I D H 2$ hotspot mutations were also not identified in 12 mucinous (29) or 316 serous ovarian carcinomas (9). These combined results suggested that IDHI and IDH2 potential hotspot mutations may not be common in patients with ovarian carcinoma.

Among these patients, the POLE1 mutation has been previously found to be frequent in 37 ovarian endometrioid carcinomas (26), whereas ring finger protein 43 (RNF43) mutations were recurrent in 15 mucinous ovarian carcinomas (25) (Table I). In the present study, neither endometrioid nor mucinous ovarian carcinomas were detected to harbor DICER1, CTCF, RPL22, DNMT3A, TRRAP, IDH1 and IDH2 hotspot mutations. These results suggested that these potential hotspot mutations observed in other (sub)types of cancer may not play synergistic roles with POLE1 or RNF43 mutations in the carcinogenesis of endometrioid or mucinous ovarian carcinomas, respectively.

The main limitation of the present study was that only short DNA fragments spanning the potential hotspot mutations were screened in the seven genes, and therefore, there is a possibility that mutations in other residues of these genes may exist in these samples. However, due to the shortage of DNA amounts, this hypothesis was not tested.

In conclusion, DICER1, CTCF, RPL22, DNMT3A, TRRAP, $I D H 1$ and $I D H 2$ hotspot mutations were not identified in 251 Chinese patients with diverse subtypes of ovarian carcinoma. These results were generally consistent with previous studies and these combined results indicated that the hotspot mutations in these genes may not be actively involved in the carcinogenesis of Chinese patients with ovarian carcinoma, except for DICER1 mutations in Sertoli-Leydig cell tumors.

\section{Acknowledgements}

The present study was supported by grants from the National Natural Science Foundation of China (nos. 81260384 and 81260097) and the Natural Science Foundation of Jiangxi Province (no. 20114BAB215033).

\section{References}

1. Parsons DW, Jones S, Zhang X, et al: An integrated genomic analysis of human glioblastoma multiforme. Science 321: 1807-1812, 2008.

2. Wu J, Jiao Y, Dal Molin M, et al: Whole-exome sequencing of neoplastic cysts of the pancreas reveals recurrent mutations in components of ubiquitin-dependent pathways. Proc Natl Acad Sci USA 108: 21188-21193, 2011

3. Heravi-Moussavi A, Anglesio MS, Cheng SW, et al: Recurrent somatic DICER1 mutations in nonepithelial ovarian cancers. N Engl J Med 366: 234-242, 2012.

4. Ley TJ, Ding L, Walter MJ, et al: DNMT3A mutations in acute myeloid leukemia. N Engl J Med 363: 2424-2433, 2010.

5. Tefferi A, Lasho TL, Abdel-Wahab O, et al: IDH1 and IDH2 mutation studies in 1473 patients with chronic-, fibrotic- or blast-phase essential thrombocythemia, polycythemia vera or myelofibrosis. Leukemia 24: 1302-1309, 2010.

6. Ogino S, Liao X, Imamura Y, et al: Predictive and prognostic analysis of PIK3CA mutation in stage III colon cancer intergroup trial. J Natl Cancer Inst 105: 1789-1798, 2013.

7. Bast RC Jr, Hennessy B and Mills GB: The biology of ovarian cancer: new opportunities for translation. Nat Rev Cancer 9: 415-428, 2009.

8. Cho KR and Shih IeM: Ovarian cancer. Annu Rev Pathol 4: 287-313, 2009.

9. Cancer Genome Atlas Research Network: Integrated genomic analyses of ovarian carcinoma. Nature 474: 609-615, 2011.

10. Wiegand KC, Shah SP, Al-Agha OM, et al: ARID1A mutations in endometriosis-associated ovarian carcinomas. N Engl J Med 363: 1532-1543, 2010.

11. Rafnar T, Gudbjartsson DF, Sulem P, et al: Mutations in BRIP1 confer high risk of ovarian cancer. Nat Genet 43: 1104-1107, 2011.

12. Loveday C, Turnbull C, Ramsay E, et al: Germline mutations in RAD51D confer susceptibility to ovarian cancer. Nat Genet 43: 879-882, 2011

13. Jones S, Wang TL, Shih IeM, et al: Frequent mutations of chromatin remodeling gene ARID1A in ovarian clear cell carcinoma. Science 330: 228-231, 2010.

14. Romero I, Sun CC, Wong KK, Bast RC Jr and Gershenson DM: Low-grade serous carcinoma: new concepts and emerging therapies. Gynecol Oncol 130: 660-666, 2013.

15. Martini M, Vecchione L, Siena S, Tejpar S and Bardelli A: Targeted therapies: how personal should we go? Nat Rev Clin Oncol 9: 87-97, 2011.

16. Yoshida K, Toki T, Okuno Y, et al: The landscape of somatic mutations in Down syndrome-related myeloid disorders. Nat Genet 45: 1293-1299, 2013.

17. Zighelboim I, Mutch DG, Knapp A, et al: High frequency strand slippage mutations in CTCF in MSI-positive endometrial cancers. Hum Mutat 35: 63-65, 2014.

18. Novetsky AP, Zighelboim I, Thompson DM Jr, Powell MA, Mutch DG and Goodfellow PJ: Frequent mutations in the RPL22 gene and its clinical and functional implications. Gynecol Oncol 128: 470-474, 2013.

19. Wei X, Walia V, Lin JC, et al: Exome sequencing identifies GRIN2A as frequently mutated in melanoma. Nat Genet 43: 442-446, 2011.

20. Yan H, Parsons DW, Jin G, et al: IDH1 and IDH2 mutations in gliomas. N Engl J Med 360: 765-773, 2009.

21. Abbas S, Lugthart S, Kavelaars FG, et al: Acquired mutations in the genes encoding IDH1 and IDH2 both are recurrent aberrations in acute myeloid leukemia: prevalence and prognostic value. Blood 116: 2122-2126, 2010.

22. Watanabe T, Nobusawa S, Kleihues P and Ohgaki H: IDH1 mutations are early events in the development of astrocytomas and oligodendrogliomas. Am J Pathol 174: 1149-1153, 2009.

23. Im AP, Sehgal AR, Carroll MP, et al: DNMT3A and IDH mutations in acute myeloid leukemia and other myeloid malignancies: associations with prognosis and potential treatment strategies. Leukemia 28: 1774-1783, 2014.

24. Kihara R, Nagata Y, Kiyoi H, et al: Comprehensive analysis of genetic alterations and their prognostic impacts in adult acute myeloid leukemia patients. Leukemia 28: 1586-1595, 2014.

25. Zou Y, Wang F, Liu FY, et al: RNF43 mutations are recurrent in Chinese patients with mucinous ovarian carcinoma but absent in other subtypes of ovarian cancer. Gene 531: 112-116, 2013. 
26. Zou Y, Liu FY, Liu H, et al: Frequent POLE1 p.S297F mutation in Chinese patients with ovarian endometrioid carcinoma. Mutat Res Fundam Mol Mech Mutagen 761: 49-52, 2014.

27. Zou Y, Zeng Y, Zhang DF, Zou SH, Cheng YF and Yao YG: IDH1 and IDH2 mutations are frequent in Chinese patients with acute myeloid leukemia but rare in other types of hematological disorders. Biochem Biophys Res Commun 402: 378-383, 2010

28. Witkowski L, Mattina J, Schonberger S, et al: DICER1 hotspot mutations in non-epithelial gonadal tumours. Br J Cancer 109: 2744-2750, 2013.

29. Ryland GL, Hunter SM, Doyle MA, et al: RNF43 is a tumour suppressor gene mutated in mucinous tumours of the ovary. J Pathol 229: 469-476, 2013.

30. Cancer Genome Atlas Research Network, Kandoth C, Schultz N, Cherniack $\mathrm{AD}$, et al: Integrated genomic characterization of endometrial carcinoma. Nature 497: 67-73, 2013.

31. Murugan AK, Yang C and Xing M: Mutational analysis of the GNA11, MMP27, FGD1, TRRAP and GRM3 genes in thyroid cancer. Oncol Lett 6: 437-441, 2013.

32. Parry M, Rose-Zerilli MJ, Gibson J, et al: Whole exome sequencing identifies novel recurrently mutated genes in patients with splenic marginal zone lymphoma. PLoS One 8: e83244, 2013.
33. Yan XJ, Xu J, Gu ZH, et al: Exome sequencing identifies somatic mutations of DNA methyltransferase gene DNMT3A in acute monocytic leukemia. Nat Genet 43: 309-315, 2011.

34. Neumann M,Heesch S, Schlee C, et al: Whole-exome sequencing in adult ETP-ALL reveals a high rate of DNMT3A mutations. Blood 121: 4749-4752, 2013.

35. Walter MJ, Ding L, Shen D, et al: Recurrent DNMT3A mutations in patients with myelodysplastic syndromes. Leukemia 25: 1153-1158, 2011

36. Kim MS, Kim YR, Yoo NJ and Lee SH: Mutational analysis of DNMT3A gene in acute leukemias and common solid cancers. APMIS 121: 85-94, 2013.

37. Bleeker FE, Lamba S, Leenstra S, et al: IDH1 mutations at residue p.R132 (IDH1(R132)) occur frequently in high-grade gliomas but not in other solid tumors. Hum Mutat 30: 7-11, 2009

38. Kang MR, Kim MS, Oh JE, et al: Mutational analysis of IDH1 codon 132 in glioblastomas and other common cancers. Int J Cancer 125: 353-355, 2009.

39. Mauzo SH, Lee M, Petros J, et al: Immunohistochemical demonstration of isocitrate dehydrogenase 1 (IDH1) mutation in a small subset of prostatic carcinomas. Appl Immunohistochem Mol Morphol 22: 284-287, 2014. 